\title{
Multiple Sclerosis: It's Not The Disease You Thought It Was
}

\author{
Loren A. Rolak, M.D, Department of Neurology, Marshfield Clinic, Marshfield, Wisconsin
}

[See related article: 61 - 62]

\section{REPRINT REQUESTS:}

Loren A. Rolak, MD

Department of Neurology

Marshfield Clinic

1000 North Oak Avenue

Marshfield, WI 54449

Telephone: 715-387-5511

Fax: 715-387-5240

Email: rolak.Ioren@marshfieldclinic.org

\section{KEYWORDS:}

Multiple Sclerosis; Nervous system diseases; Demyelinating diseases; Interferons

RECEIVED:

June 6, 2002

REVISED AND ACCEPTED:

September 4, 2002

Clinical Medicine \& Research

Volume 1, Number 1: 57 - 60

(C2003 Clinical Medicine \& Research

www.mfldclin.edu/clinmedres
Multiple sclerosis (MS), the most common disabling neurologic disease of young people, afflicts approximately a quarter of a million Americans. The symptoms of MS result from recurrent attacks of inflammation in the central nervous system, which probably occur through an autoimmune mechanism. The target of the immune attack is myelin, the lipoprotein sheath that surrounds the axons and insulates them, and enhances nerve conduction. The white matter of the brain takes its name from the glistening white appearance of this lipid wrapping, which contains most of the pathways, tracts and axonal projections of the central nervous system. (The gray matter contains primarily the cell bodies of the neurons themselves.) Myelin is made by cells called oligodendrocytes and when it is inflamed and damaged, nerve conduction is disrupted and nerves thus lose function, thereby producing the neurologic symptoms of MS.

\section{PATHOPHYSIOLOGY}

The cause of MS is unknown and its pathophysiology remains poorly understood. Patients are not born with MS, but rather some environmental factor apparently acts on genetically susceptible individuals to produce the disease; but the nature of that factor (such as whether or not it is a virus) remains elusive. The best accepted explanation postulates that macrophages present myelin antigens to appropriate T-cells, thereby activating the T-cells to proliferate. They then cross the blood-brain barrier through interactions with intercellular adhesion molecules and once inside the central nervous system, they release cytokines that further damage myelin and that perpetuate the immune response. The details of this process, including the nature of the triggering antigen, are still subject to speculation but there is strong evidence that MS is a T-cell mediated autoimmune attack on the central nervous system. Concomitant with the myelin destruction, there is also damage to the underlying axon, which leads to further disability. ${ }^{1}$

Among the unanswered questions is whether MS is a single disease. It could be that different antigens are involved in different patients, different T-cells are activated, or the mechanisms of cell damage are different. ${ }^{2}$

\section{EPIDEMIOLOGY}

Multiple sclerosis favors women over men by a ratio of nearly 2 to 1 , and it strikes most often between the ages of 20 and 40 . Caucasians are especially vulnerable, particularly those of northern European extraction, and there is a geographic preference for people living in northern latitudes. Though clearly not inherited in a simple Mendelian pattern, MS tends to cluster slightly within families, as there is a 1 to $5 \%$ risk of developing MS if a parent or sibling has the disease, and at least a $25 \%$ concordance among monozygotic twins. 


\section{CLINICAL FEATURES}

Variability and diversity characterize the symptoms and presentation of MS. There is virtually no neurologic complaint that has not been traced to MS at one time or another, and a comprehensive account of its clinical features can become nothing more than a mere recitation of a positive neurologic review of systems. The most common symptoms are listed in table 1. Symptoms that arise directly from damage to neurons (that is to say, gray matter symptoms) occur so rarely that their appearance casts doubt on the diagnosis of MS. Examples of such gray matter symptoms are listed in table $2 .{ }^{3}$

Most symptoms develop abruptly, within hours or days. These attacks or relapses of MS typically reach their peak within a few days at most and then resolve slowly over the next several days or weeks so that a typical relapse will be symptomatic for about eight weeks from onset to recovery. Resolution is often complete. However, the pattern of presentation, like so many features of MS, is highly variable and symptoms may fluctuate considerably or even progress with little resolution. Attacks strike approximately every 12 to 18 months. This pattern is common when patients first develop MS and through the early years of their disease, and is referred to as relapsing-remitting MS. In many patients, over a span of 5 to 15 years, the attacks begin more indolently, persist more chronically and remit less completely, gradually transforming into a pattern of steady deterioration rather than episodic flares. This pattern is referred to as secondary progressive MS. The pathophysiology responsible for this transformation from a relapsing disease into a progressive one is poorly understood but has important implications for treatment as many of the drugs effective for preventing relapses seem useless in the secondary progressive phase of the disease.

\section{PROGNOSIS}

Multiple sclerosis is seldom fatal and life expectancy is shortened by only a few months. Concerns about prognosis center primarily on the quality of life and prospects for disability. Most patients and physicians harbor an unfounded view of MS as a relentlessly progressive, inevitably disabling disease. The truth is that 15 years after the onset of MS, only about $20 \%$ of patients are bedridden or institutionalized. Another $20 \%$ may require a wheelchair, or use crutches, or a cane to ambulate, but fully $60 \%$ will be ambulatory without assistance and some will have little deficit at all. Perhaps as many as $1 / 3$ of all patients with MS go through life without any persistent disability, and suffer only intermittent, transient episodes of symptoms.

\section{DIAGNOSIS}

MS can be among the most difficult of all diseases to diagnose because of the bewildering number of symptoms it causes and the multiple ways in which they can present. The "typ- ical" MS patient is a young woman with abrupt, focal neurologic symptoms occurring discretely or in combinations, lasting weeks to months and then resolving, with new or recurrent symptoms developing months to years later. The diagnosis may be especially difficult, or indeed impossible, when the patient is older, when symptoms are strictly progressive, or when there has been only one episode of neurologic dysfunction. Tests can buttress the clinical diagnosis of MS, but no laboratory findings are pathognomonic and all tests have pitfalls that limit their usefulness.

Magnetic resonance imaging (MRI) is a very sensitive but disappointingly non-specific technique for visualizing the inflammatory lesions of MS, which appear as multiple, irregular, confluent areas of increased signal intensity within the white matter of the brain, particularly around the ventricles. Nearly $90 \%$ of patients with MS have abnormal MRI scans. Various analyses and algorithms have shown that an MRI of the head should be the first test ordered to evaluate suspected MS patients. The major disadvantage of MRI remains its lack of specificity, since many conditions mimic MS on MRI. Too frequently, these "false positives" often inappropriately label patients with the diagnosis of MS and over-diagnosis of MS based on MRI changes (figure 1).

Abnormalities in the cerebrospinal fluid (CSF) are sufficiently common and characteristic to make CSF analysis fairly accurate for the diagnosis of MS. Spinal fluid protein and white blood cell counts are occasionally mildly elevated, but the most useful findings are the increases in the immunoglobulin $\mathrm{G}(\mathrm{IgG})$ level and synthesis rate. Immunoglobulins in the spinal fluid, presumably reflecting the underlying autoimmune activation, appear as distinct oligoclonal bands on CSF electrophoresis. The pattern formed by these bands varies from patient to patient, but they are present in some form in approximately $90 \%$ of all MS patients, while the few other diseases that produce similar banding are seldom mistaken for MS. The major obstacle to the use of CSF for the diagnosis of MS is the reluctance of patients to undergo lumbar puncture.

Table 1. Most common symptoms of MS.

1. Weakness or numbness in one or more limbs.

2. Optic neuritis: Painful monocular visual loss.

3. Tremor and ataxic gait from cerebellar dysfunction.

4. Double vision, dysarthria, or dizziness from brainstem dysfunction. 5. Fatigue.

Table 2. Symptoms unlikely to be caused by MS.

1. Dementia.

2. Aphasia.

3. Loss of consciousness: seizures of syncope.

4. Pain.

5. Muscle atrophy or fasciculations.

58 CM\&R 2003: 1 (January) Rolak 
Evoked potentials play a more limited role in diagnosing MS. Evoked potentials measure conduction along specific central nervous system pathways by recording the electroencephalographic response to visual, auditory, or sensory stimulation.
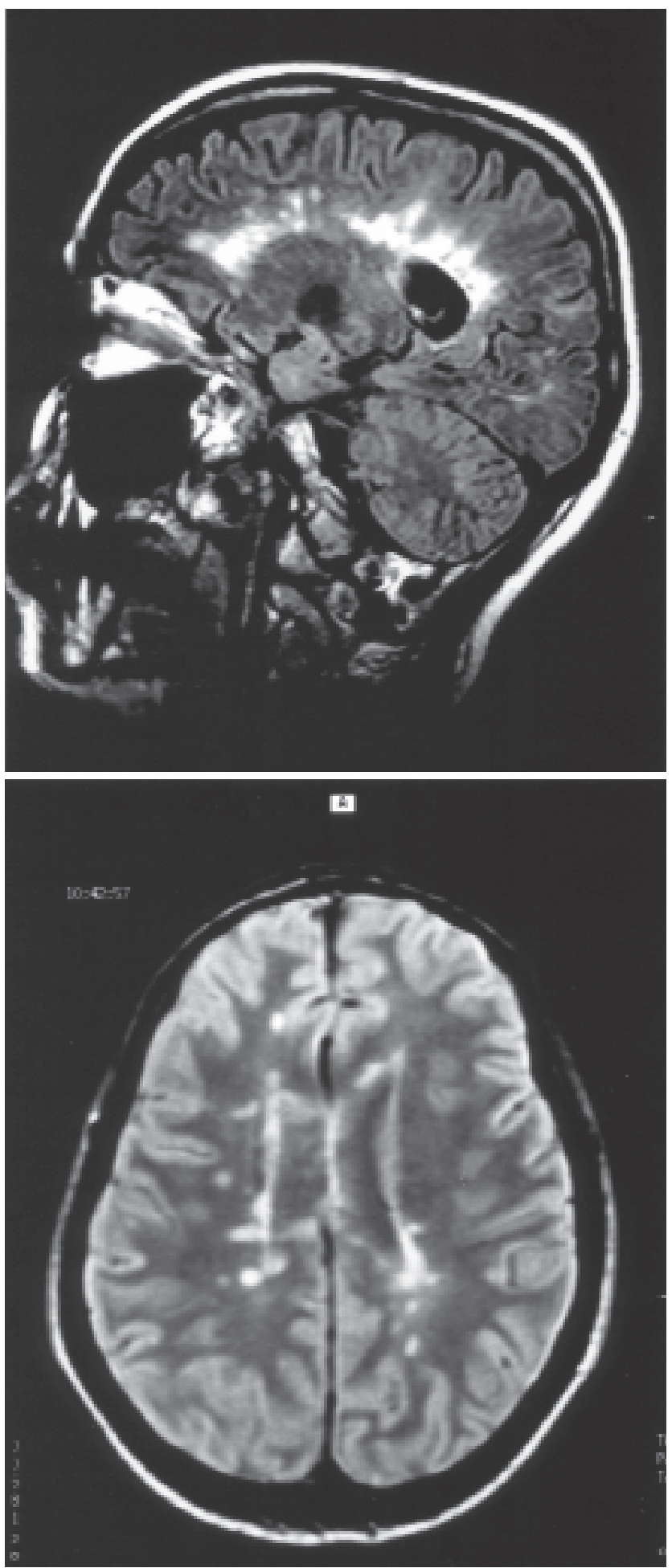

Figure 1. Axial T2-weighted MRI scan of the brain showing characteristic lesions of MS. These are irregular, primarily periventricular signal changes. The sagittal FLAIR scan shows the typical anatomy of the MS plaques radiating vertically away from the ventricles.
A slowing in conduction is presumed to reflect inflammation and demyelination in that pathway, thus detecting an asymptomatic MS lesion. The sensitivity and specificity of evoked potentials do not approach those of the MRI or CSF but they sometimes uncover unsuspected lesions and thereby heighten the probability of MS.

The list of medical conditions that can cause multi-focal neurologic problems in young people is quite extensive and so the differential diagnosis of MS is far ranging. Table 3 is a non-exhaustive list of some of the more common conditions that mimic MS.

Traditionally, the diagnosis of MS depends upon showing that there is sclerosis (scarring or inflammation) that is multiple-patients must have two separate CNS lesions that have occurred in two or more separate episodes, which is to say they must have lesions disseminated in space and in time. These must cause white matter symptoms not gray matter symptoms. The neurological examination should show these objective abnormalities (preferably in a young patient between the ages of 20 and 40). Importantly, there should be no other disease accounting for the symptoms. In addition to these clinical criteria, the diagnosis can be supported using laboratory testing such as MRI scans, CSF analysis and evoked potentials. There nevertheless remain many pitfalls and nuances in the diagnosis of MS, and ultimately physicians often rely on their own judgment to diagnose MS rather than relying on predetermined or "official" criteria. 4

Many patients who suffer an isolated monosymptomatic episode of demyelination, such as optic neuritis or transverse myelitis, will ultimately develop a second inflammatory event and so will be diagnosed as having MS. This is particularly true if MRI scanning of the brain at the time of their initial demyelinating event reveals white matter changes characteristic of MS. Therefore, patients with a single episode of demyelination and abnormal MRI scan of the brain are often presumed to be suffering from MS already. (This is true even though most "official" diagnostic criteria would not classify these patients as definite MS.) There remain some cases of clinically isolated syndromes with normal head MRI's who apparently never develop clinically definite MS.

Table 3. Partial differential diagnosis of MS.

1. Hysteria and somatization disorders.

2. Postviral demyelination (acute disseminated encephalomyelitis).

3. Vasculitis affecting the CNS.

4. Spino-cerebellar degenerations.

5. Lyme disease.

6. Neurosarcoidosis.

7. Stroke in the young.

8. Inherited white matter diseases (leukodystrophies).

9. Non-specific MRI abnormalities. 


\section{ACUTE THERAPY}

Acute relapses of MS are usually treated with corticosteroids. There are few good controlled studies of the effectiveness of steroids, the optimum dosage, route of administration, duration of treatment, or most appropriate indications for their use. Nevertheless, steroid treatments have become a traditional and accepted standard of practice for new attacks of MS and there is a universal sense that they shorten symptoms, enhance well-being and provide many benefits for acute relapses. A standard regimen uses intravenous methylprednisolone (Solumedrol) 1 gram daily for three to five days, sometimes (but not always, depending on physician preference) followed by a tapering dose of oral steroids. Steroids reduce inflammation, seal the blood/brain barrier, enhance nerve conduction and alter the immune system, all of which are potentially beneficial in treating MS. Whatever benefits they have seem to be limited primarily to acute attacks and it is less clear that they can fundamentally alter the natural history of MS or prevent ultimate disability.

\section{DISEASE MODIFYING THERAPY}

Five drugs are currently approved by the Food and Drug Administration as disease modifying agents that alter the natural history of relapsing-remitting MS. The four self-administered drugs are intramuscular beta-interferon-la (Avonex), subcutaneous beta-interferon-la (Rebif), subcutaneous betainterferon-lb (Betaseron), and glatiramer acetate (Copaxone). These medications all reduce the number of attacks in relapsing-remitting MS. They seem to have little effect once the disease has entered a secondary progressive phase. The mechanism of action of these drugs is unknown, but the interferons probably induce secretion of a variety of immunomodulatory proteins while glatiramer probably inhibits the activation of myelin reactive T-cells. ${ }^{5}$

Avonex is a preparation of recombinant human beta-interferon-la, administered in a dosage of 6 million IU intramuscularly once a week. Rebif is an identical preparation, administered as 12 million IU subcutaneously three times a week. Betaseron is recombinant beta-interferon-lb, which differs from Avonex and Rebif only in some minor alterations of a single amino acid substitution and less glycosolation, though it is doubtful that these changes make it truly a different drug. It is given as 12 million IU subcutaneously every other day. Copaxone differs from all these drugs because it is not an interferon but rather is a synthetic polypeptide composed of sequences of four amino acids that share many antigenic similarities to myelin basic protein and appears to alter the immune response to myelin. It is a subcutaneous preparation given daily. There are still no definitive head-to-head comparisons of these drugs and so the differences among them (such as whether higher doses of interferons confer greater benefits) are still a matter of conjecture and debate. At present, these four drugs can be considered approximately equal in efficacy and therapeutic decisions are often made based on convenience of dosage, route of administration, side effects, concern for neutralizing antibodies (which develop against the interferons after about a year in 2 to $30 \%$ of patients) and similar practical issues. Although these medications have some benefits in minimizing the rate of attacks, it is not known whether they will delay the accumulation of neurologic deficits and so postpone disability. Their long-term benefits are thus unclear. ${ }^{6}$

For secondary progressive MS, the most convincing data favors mitoxantrone (Novantrone) as most likely to retard progression and delay disability. Novantrone is a well-established cancer chemotherapeutic drug, primarily effective for lymphomas and leukemias, with broad immune-altering properties. It can delay the accumulation of disability in patients with secondary progressive MS when administered in a dosage of, $12 \mathrm{mg} / \mathrm{m}^{2}$ intravenously every three months. Such periodic treatments can slow the progression of MS but, when a cumulative dose of $100-140 \mathrm{mg} / \mathrm{m}^{2}$ has been given, the risk of irreversible toxicity from the drug precludes further administration. The primary toxicity is myocardial damage, and so echocardiograms and ejection fractions are followed periodically throughout treatment. For most patients, toxicity limits treatment to a duration of only two or three years and so Novantrone should be considered a short-term treatment option.

\section{CONCLUSION}

In just the past few years, research has further clarified the cellular and molecular events that accompany an MS immune attack on myelin and has raised speculation that there could be several diseases comprising what we now call MS. Epidemiological studies have also clarified the prognosis and reaffirmed that many patients do well. The distinction between relapsing-remitting MS and secondary progressive MS has assumed increasing importance, in part because of different responses to treatment. New criteria for the diagnosis of MS have also been developed to take advantage of the extraordinary sensitivity of MR imaging. Most importantly, in less than 10 years, five new drugs have been developed with proven ability to alter the natural history of MS and mitigate the disease. These advances have completely altered the clinician's approach to the patient with MS and foreshadow new hope for the ultimate conquest of this disease.

\section{REFERENCES}

1. Rolak L. Multiple Sclerosis. In: Rolak L, Harati Y. (Eds.): Neuroimmunology for the Clinician. Boston: Butterworth-Heineman, 1997, pp 107-132.

2. Lucchinetti C, Buck W, Parisi J, Scheithauer B, Rodriguez M, Lassmann H. Heterogeneity of multiple sclerosis lesions: implications for the pathogenesis of demyelination. Ann Neurol 2000; 47: 707-717.

3. Compston A, Ed.: McAlpine's Multiple Sclerosis. 3rd Edition. London: Churchil-Livingstone, 1998.

4. McDonald WI, Compston A, Edan G, et al. Recommended diagnostic criteria for multiple sclerosis: guidelines from the international panel on the diagnosis of MS. Ann Neurol 2001; 50:121-127.

5. Dhib-Jalbut S. Mechanisms of action of interferons and glatiramer acetate in multiple sclerosis. Neurology 2002; 58 (Suppl 4): 3-9.

6. Calabresi PA. Considerations in the treatment of relapsing-remitting multiple sclerosis. Neurology 2002; 58 (Suppl. 4): 10-22. 